\title{
Simulating Uncertainty of Early Warning Scores in Sepsis Detection
}

\author{
Ali Jazayeri \\ College of Computing and \\ Informatics, Drexel University \\ aj629@drexel.edu
}

Siddhartha Nambiar

Department of Industrial and

Systems Engineering, North

Carolina State University

snambia@ncsu.edu

\author{
Muge Capan \\ Decision Sciences \& MIS Department, LeBow \\ College of Business, Drexel University \\ mc3922@drexel.edu
}

Department of Industrial and

Systems Engineering, North

Carolina State University

memayorg@ncsu.edu

\author{
Julie Ivy \\ Department of Industrial and \\ Systems Engineering, North \\ Carolina State University \\ jsivy@ncsu.edu
}

\author{
Christopher Yang \\ College of Computing and \\ Informatics, Drexel University \\ chris.yang@drexel.edu
}

\author{
Ryan Arnold \\ College of Medicine, \\ Hahnemann University \\ Hospital, Drexel University \\ rca57@drexel.edu
}

\begin{abstract}
Sepsis, a syndrome of acute organ dysfunction secondary to a systemic infection, has been recognized as a growing burden on healthcare systems and is responsible for nearly $\$ 24$ billion annually. Various early sepsis detection scores have been developed to quantify the risk of clinical deterioration. While the scores quantify risk, they often overlook the uncertainties associated with the scores, defined as a feature of a scoring system caused by any absence of a component in documented Electronic Health Records (EHR). Using a discrete-event simulation approach, we analyzed the simulated 1) composition of a selected early warning score, 2) timing of worst and best score values of sepsis population in the Emergency Department, and 3) changes over time in uncertainty throughout the hospitalization. Composition referred to simulated availability of score components and uncertainty was represented by the percent of components that are unavailable at a given time point. Data was collected from 15,982 patients with 22,968 visits meeting infection and sepsis criteria within subpopulations with different levels of severity of sepsis. Simulated trajectories indicated differences among the sub-populations. The first complete score and worst score values were found to be higher for those who died with sepsis compared to those that survived with infection and/or with sepsis. The findings from this study provide a framework to capture the stochastic nature of early warning scores and can inform implementation of scores in clinical practice with the goal of supporting early detection of sepsis.
\end{abstract}

CCS CONCEPTS: • Applied computing Health informatics

KEYWORDS: Sepsis; Early Warning System; Uncertainty; Simulation; Emergency Department

\footnotetext{
Permission to make digital or hard copies of part or all of this work for personal or classroom use is granted without fee provided that copies are not made or distributed for profit or commercial advantage and that copies bear this notice and the full citation on the first page. Copyrights for third-party components of this work must be honored. For all other uses, contact the Owner/Author.

$A C M-B C B$ '19, September 7-10, 2019, Niagara Falls, NY, USA

(c) 2019 Copyright is held by the owner/author(s).

ACM ISBN 978-1-4503-6666-3/19/09.

https://doi.org/10.1145/3307339.3343252
}

\section{ACM Reference format:}

Ali Jazayeri, Muge Capan, Christopher Yang, Siddhartha Nambiar, Maria Mayorga, Julie Ivy, Ryan Arnold. 2019. Simulating Uncertainty of Early Warning Scores in Early Sepsis Detection. In The 10th ACM Conference on Bioinformatics, Computational Biology, and Health Informatics (ACM BCB). ACM, Niagara Falls, NY, USA.

https://doi.org/10.1145/3307339.3343252

\section{BIOGRAPHY}

Ali Jazayeri is a Ph.D. candidate at The College of Computing and Informatics (CCI) at Drexel University and his dissertation focuses on mining frequent substructures and their evolution in temporal networks. Ali Jazayeri is an instructor in the MS in Data Science program at CCI and he is working in the Healthcare Informatics Research Laboratory under the supervision of Prof. Chris Yang.

\section{ACKNOWLEDGMENTS}

We would like to acknowledge that this work has been performed on behalf of the S.E.P.S.I.S (Sepsis Early Prediction Support Implementation System) Collaborative. This work was supported by the National Science Foundation Smart and Connected Health (Award Number: 1833538), the National Library of Medicine of the National Institutes of Health (Grant Number: 1R01LM012300-01A1, Award Number: R01LM012300) and partially by the National Science Foundation under the Grant NSF-1741306, IIS-1650531, and DIBBs-1443019. Any opinions, findings, and conclusions or recommendations expressed in this material are those of the author(s) and do not necessarily reflect the views of the National Science Foundation.

\section{REFERENCES}

[1] Rhee, C., Dantes, R., Epstein, L., Murphy, D. J., Seymour, C. W., Iwashyna, T. J., Kadri, S. S., Angus, D. C., Danner, R. L. and Fiore, A. E. 2017. Incidence and trends of sepsis in US hospitals using clinical vs claims data, 2009-2014. Jama, $318,13,1241-1249$.

[2] Smyth, M. A., Brace-McDonnell, S. J. and Perkins, G. D. 2016. Impact of Prehospital Care on Outcomes in Sepsis: A Systematic Review. The western journal of emergency medicine, 17, 4, 427-437.

[3] Bayer, O., Hartog, C. S., Schwarzkopf, D., Stumme, C., Stacke, A., Bloos, F., Hohenstein, C., Kabisch, B., Weinmann, C., Winning, J., Sakr, Y. and Reinhart, J. K. 2014. The PRESEP score: an early warning scoring system to identify septic patients in the emergency care setting. Critical Care, 18, S2. 